\title{
A novel method to remove the background from $x$-ray diffraction signal
}

\section{Zheng, Yi; Speller, Robert; Griffiths, Jennifer}

\section{Published in:}

Physics in Medicine and Biology

Link to article, DOI:

10.1088/1361-6560/aaac9e

Publication date:

2018

Document Version

Early version, also known as pre-print

Link back to DTU Orbit

Citation (APA):

Zheng, Y., Speller, R., \& Griffiths, J. (2018). A novel method to remove the background from x-ray diffraction signal. Physics in Medicine and Biology, 63(6), [06NT03]. https://doi.org/10.1088/1361-6560/aaac9e

\section{General rights}

Copyright and moral rights for the publications made accessible in the public portal are retained by the authors and/or other copyright owners and it is a condition of accessing publications that users recognise and abide by the legal requirements associated with these rights.

- Users may download and print one copy of any publication from the public portal for the purpose of private study or research.

- You may not further distribute the material or use it for any profit-making activity or commercial gain

- You may freely distribute the URL identifying the publication in the public portal

If you believe that this document breaches copyright please contact us providing details, and we will remove access to the work immediately and investigate your claim 


\title{
A novel method to remove the background from X-ray diffraction signal
}

\author{
Yi Zheng ${ }^{1}$, Robert Speller ${ }^{1}$ and Jennifer Griffiths ${ }^{1,2}$ \\ 1 Department of Medical Physics and Biomedical Engineering, University \\ College London, Gower Street, London WC1E 6BT, UK \\ 2 UCL Faculty of Engineering Sciences, University College London, Gower \\ Street, London WC1E 6BT, UK \\ E-mail: yi.zheng.11@ucl.ac.uk
}

July 2017

\begin{abstract}
The first step that is required to extract the correct information from a twodimensional (2D) diffraction signature is to remove the background accurately. However, direct background subtraction inevitably overcorrects the signal as it does not take into account the attenuation by the sample. Other traditional background removal methods, such as the rolling ball technique, can separate sharp diffraction peaks of crystalline materials from their background. These methods are unsuitable for biological tissue, which is amorphous and does not have sharp diffraction peaks. This technical note proposes a novel method that combines peak fitting and experimental results to estimate the background for 2D XRD signals.
\end{abstract}

Keywords: X-ray diffraction, background removal, 2D XRD

PACS: 87.59.-e X-ray imaging

Submitted to Physics in Medicine and Biology

\section{Introduction}

X-ray diffraction (XRD) has long been used for crystalline material characterisation because it reveals atomic and sub-atomic structure. It can also be used for biological tissue classification (Lewis et al 2003; Petsche and Pinsky 2013; Theodorakou and Farquharson 2009), especially breast tissue classification (Pani et al 2010; Sidhu et al 2011). In a two-dimensional (2D) XRD measurement, the background consists of the primary X-ray beam and scattering from the equipment, rather than the sample, and needs to be removed to reveal the XRD signal (Bohndiek et al 2008; Chaparian et al 2010; O’Flynn et al 2013; Poulsen 2004).

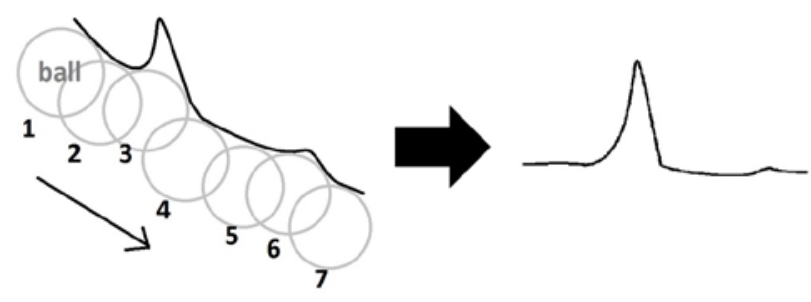

Figure 1. Schematic of the rolling ball algorithm 
For crystalline material, the background can be removed by using a 'rolling ball' algorithm (Sternberg 1983). This algorithm as demonstrated in figure 1, can be understood as pressing and rolling a virtual 'ball' from underneath the intensity surface of the XRD pattern: the background is the highest point that is reached by the 'ball' at each pixel. The algorithm can remove the amorphous signal in a XRD pattern in order to extract the diffraction signal of crystalline material (He 2009), but it cannot be used to remove the background from the diffraction of an amorphous material. Instead of being a sharp peak as the XRD signal of a crystal, the diffraction signal of an amorphous material is blurred into a wide range of angles due to the lack of long range order. Since this traditional method, the 'rolling ball' algorithm, is designed to remove any small change in gradient, but to keep the large one, the broad peak from an amorphous material will be identified by the algorithm as part of the background and thus removed.

Direct subtraction is a common method for background removal for amorphous material. The background image is an X-ray image from the system without the presence of the sample. In order to better distinguish the background image from the real background signal, from here on, the background image obtained by this method is called a flat image in this paper. By subtracting this flat image, the true 2D XRD signal can be extracted (Bohndiek 2008, O’Flynn 2013). However, this method of estimating the background level is only suitable if we can ignore the attenuation of the X-ray beam by the sample (Poulsen 2004). In other words, this only applies to a very thin and light sample, such as thin film.

Without a beam-stop in the experimental setup, the flat image contains the primary X-ray beam and scattering from the equipment (Bohndiek 2008; Sampath et al 2012). The primary beam is attenuated by the sample, and thus a direct subtraction is not suitable if the sample thickness is not negligible. One approach to correcting for this would be to divide the flat image by a single attenuation correction factor. However, as Compton scattered photons have lower energy at a larger scatter angle, the attenuation correction of the flat image should not be a single value but should have angular dependence. Even with a beam-stop, scatter from the equipment can still bypass the beam-stop as it is coming from various angles due to multiple scattering. This effect becomes even more severe when the apertures of the collimators are larger as this allows X-rays (both primary and scattering) to pass through from a wider range of angles. When using an X-ray lab source, to increase the intensity of the XRD signal, often, a large collimator aperture is chosen to allow high X-ray intensity.

In conclusion, for clinical use of XRD, a large collimator and thick sample is inevitable, and so a direct subtraction of the flat image cannot accurately correct the XRD single. Therefore, the first task to make XRD tissue diffraction suitable for clinical use is to develop a method that will remove this background signal for thick samples and large collimator apertures.

\section{Methods and results}

\subsection{Experimental method for estimating the background signal}




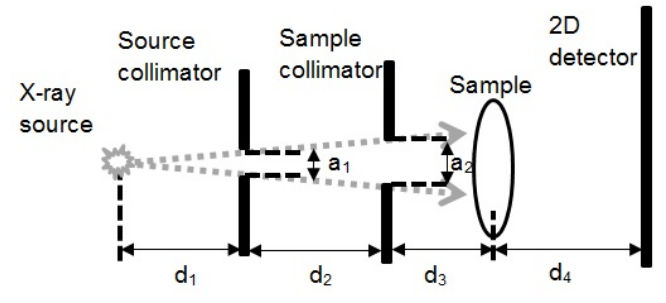

(a)

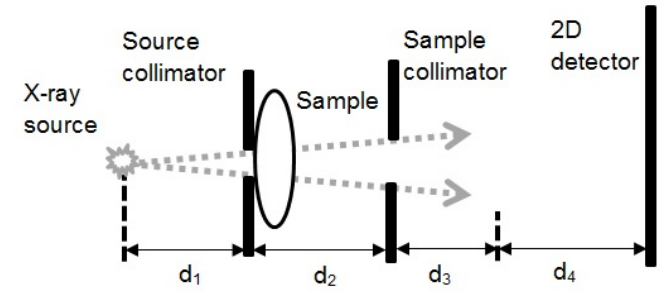

(b)

Figure 2. (a) The schematic of the experimental setup, $a_{1}=1.0 \mathrm{~mm}, \mathrm{a}_{2}=1.2 \mathrm{~mm}, \mathrm{~d}_{1}=39 \mathrm{~mm}, \mathrm{~d}_{2}=12 \mathrm{~mm}, \mathrm{~d}_{3}=2 \mathrm{~mm}, \mathrm{~d}_{4}=106.5$ $\mathrm{mm}$; (b) Position of the sample proposed in this paper to produce the true background signal.

The experimental setup is as shown in figure 2(a). The X-ray source for this work is a Molybdenum source at $30 \mathrm{kVp}$ with a $0.3 \mathrm{~mm}$ thick Molybdenum filter. The detector is a wafer-scale CMOS APS sensor coupled to a $150 \mu \mathrm{m}$ thick CsI: TI scintillator (Konstantinidis et al 2012, Zhao et al 2015) The sample is a $6 \mathrm{~mm}$ thickness of Perspex. This has a similar diffraction pattern to healthy breast tissue, and thus can be used as a tissue equivalent material for testing X-ray diffraction systems for breast tissue (Chaparian et al 2012; Zheng et al 2015). The incident beam collimation is made up of two apertures, called the source collimator and sample collimator in this work. The 2D XRD patterns were then reduced to a $1 \mathrm{D}$ XRD signal by averaging the $2 \mathrm{D}$ images radially from the center of the $2 \mathrm{D}$ XRD patterns, while this center was manually selected. As shown in figure 3 , the values of the pixels which have the same distance from the center were averaged, which can then give a diffraction pattern as a function of the distance from the center (r). By combining the geometry of the experimental setup, we can then calculate the scatter angle.
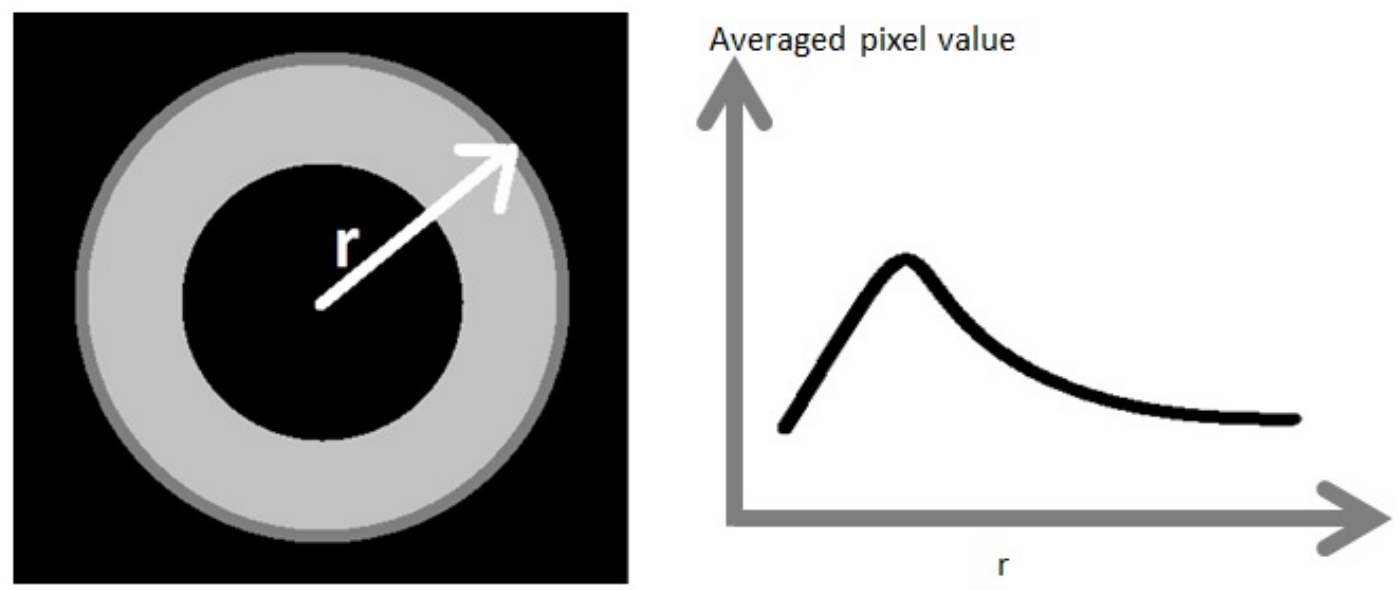

Figure 3. Schematic of the radial averaging process

The radially averaged XRD profile is defined as $I_{s}$, while the radial profile of the flat image (obtained without the sample present) is represented as $I_{f}$ in this work. Traditionally, for a small sample, the XRD signal of a material can be obtained by finding $I_{s}-I_{f}$. In this paper, we propose to place the sample between the two collimators (as shown in figure 2 (b)) and record the resulting image, $I_{b s}$ as a replacement to $I_{f}$, the flat image. The values of $I_{s}, I_{f}$ and $I_{b s}$ are shown in figure 2(a). The result 
of $I_{S}-I_{f}$ was noisy. Therefore, we smoothed the results using the moving average method with a window of 5 and plotted it in figure 4 (b).

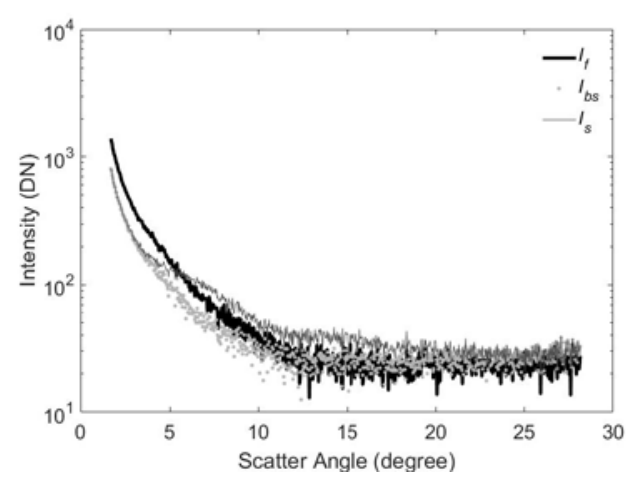

(a)

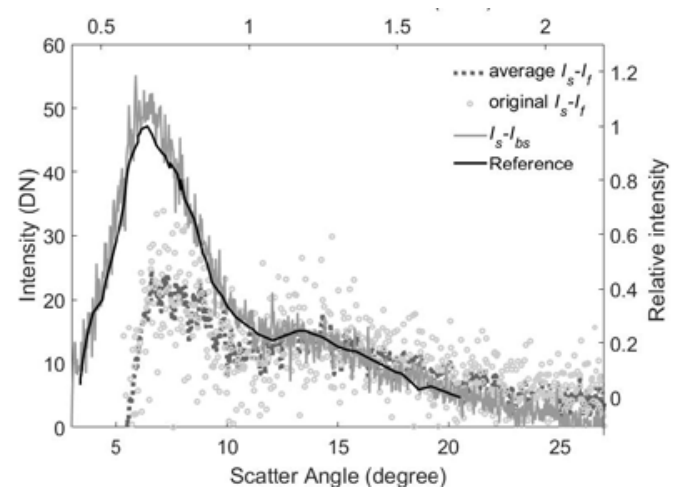

(b)

Figure 4. The values of (a) $I_{s}, I_{f}$ and $\mathrm{I}_{\mathrm{bs}}$ and (b) their subtraction (800 data points). The subtraction results (in grey) were compared to the diffraction profile of Perspex (in black) measured by Chaparian et al (2012).

As can be seen in figure $4(\mathrm{a}), I_{f}$ has a higher intensity than $I_{s}$ at scatter angles smaller than $6.5^{\circ}$. This is caused by attenuation of the beam by the sample, reducing $I_{s}$. It can be seen from figure 4 (b) that subtracting the flat image directly $\left(I_{S}-I_{f}\right)$ overcorrects the signal and results in a reduction in the diffraction peak at small angles, while subtracting $I_{b s}$ produces a XRD profile that is similar to the diffraction profile measured by Chaparian et al. (2012).

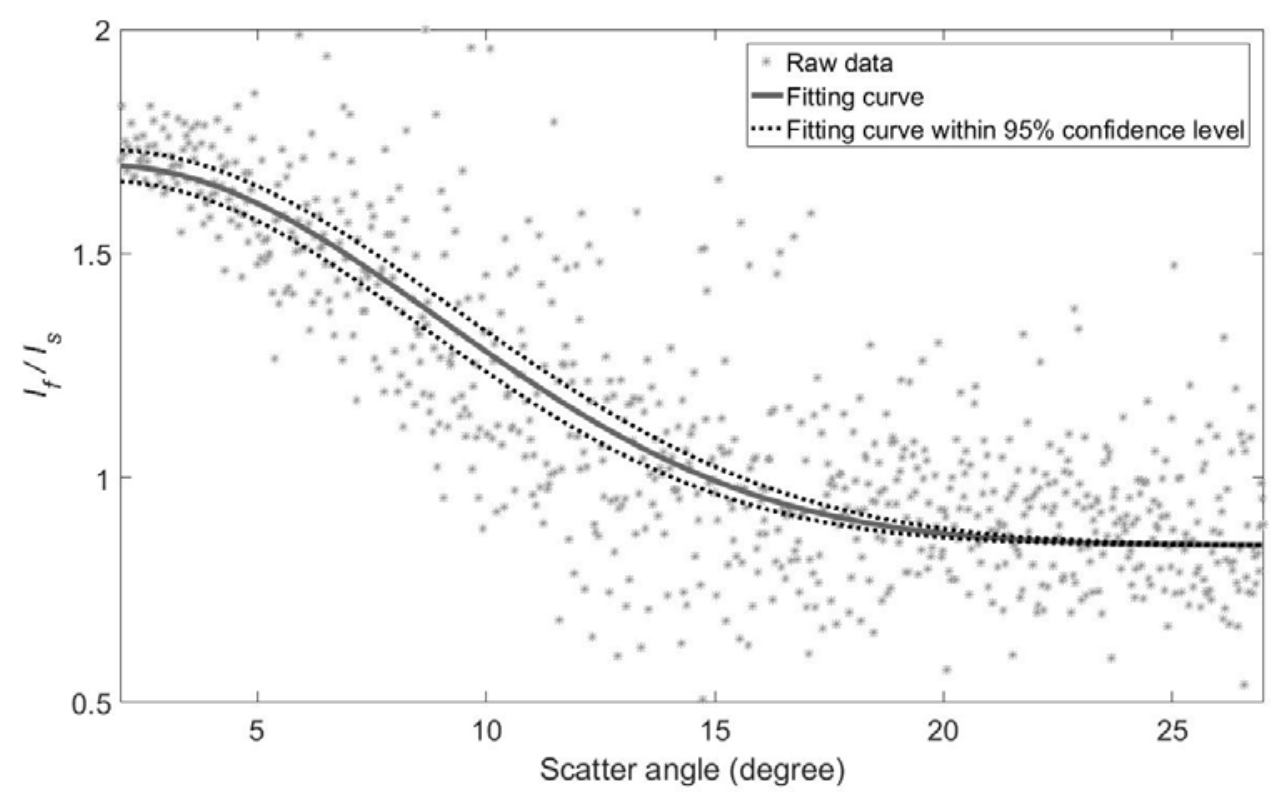

Figure 5. The ratio $I_{f} / I_{b s}$ as a function of the scatter angle. This ratio is equal to $1 / R(\theta) . R(\theta)$ represents the geometric transmission correction factor.

The X-ray focal spot usually has a Gaussian (or double-Gaussian) shape. Thus, the pinhole image of the X-ray source also has a Gaussian distribution. As $I_{f}$ and $I_{b s}$ can be understood as the radial average profiles of pinhole images, they can be approximate to a Gaussian distribution. Therefore, their quotient can also be approximate to a Gaussian distribution. To investigate the difference between 
$I_{f}$ and $I_{b s}$, the ratio of $I_{f}$ to $I_{b s}$ is plotted in figure 5 with a Gaussian fit to the data points. The fitting was done in Matlab by limiting the range of the fitting parameters so that in the following equation, $a$ is between 0.5 to 1 , b is between -0.01 and 0.01 and c is large than 0 .

$$
f(x)=a e^{-\frac{(x-b)^{2}}{2 c^{2}}}
$$

We define $1 / R(\theta)$ as $\mathrm{I}_{\mathrm{f}} / \mathrm{I}_{\mathrm{bs}}$. The ratio $1 / R(\theta)$ reflects the attenuation of the primary beam by the sample at each scatter angle. If this ratio is constant, it means that the attenuation of the primary beam is the same at different scatter angles. This is not true as scattering has angular dependence. Because this ratio is not constant, we cannot simply use $I_{f}$ multiplied by the transmission factor to correct the data. The transmission factor is given by $e^{-\mu d}$, where $\mu$ is the attenuation coefficient of the sample and $d$ is the path length of the $\mathrm{X}$-ray beam. As the scatter angle increases, the ratio $1 / R(\theta)$ decreases. This means that the beam is attenuated more at smaller scatter angles. One possible explanation could be that at small angle, the distribution of multiple scattering follows a Gaussian distribution in a forward direction (Podgorsak 2016). These scattering photos will have reduced energy compared to the original incident photons, thus can be easier absorbed by the samples. In summary, by representing $R(\theta)$ as $I_{b s}(\theta) / I_{f}(\theta)$, the true XRD signal $I(\theta)$ can be represented as:

$$
I(\theta)=I_{s}(\theta)-I_{b s}(\theta)=I_{s}(\theta)-R(\theta) I_{f}(\theta)
$$

This method requires imaging the sample twice, which results in a doubling of the radiation dose. To increase the clinical potential of XRD, we would like to reduce radiation dose as low as possible. Therefore, an alternative method is described in the following section that estimates the geometric transmission correction factor $R(\theta)$ based on $I_{s}$ and $I_{f}$. The true background signal can then be calculated from $R(\theta) I_{f}(\theta)$.

\subsection{Combination of computational and experimental methods}

As shown in figure 4(a), $I_{S}$ and $I_{b s}$ have similar values at low scatter angles $\left(<3^{\circ}\right)$ and large angles $\left(>22^{\circ}\right)$. We will define the geometric transmission correction factor, $1 / R^{\prime}(\theta)$, as equal to the Gaussian fit of $I_{f}(\theta) / I_{S}(\theta)$ for $\theta<3^{\circ}$ or $\theta>22^{\circ}$. In figure 6(a), $1 / R^{\prime}(\theta)$ is equal to $I_{f}(\theta) / I_{S}(\theta)$ while $I_{f}(\theta) / I_{b s}(\theta)=1 / R(\theta)$. The results of using this estimated correction factor $1 / R^{\prime}(\theta)$ to correct the background $I_{f}$ are shown in figure 6 (b). As can be seen from the figure, this method provides a result that is identical $(\mathrm{p}<0.01)$ to the one obtained when directly measuring the background signal $\left(I_{b s}\right)$. 


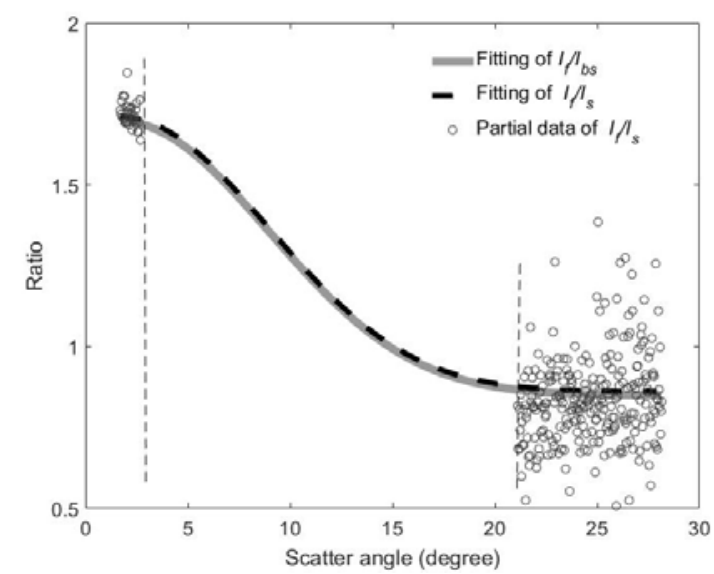

(a)

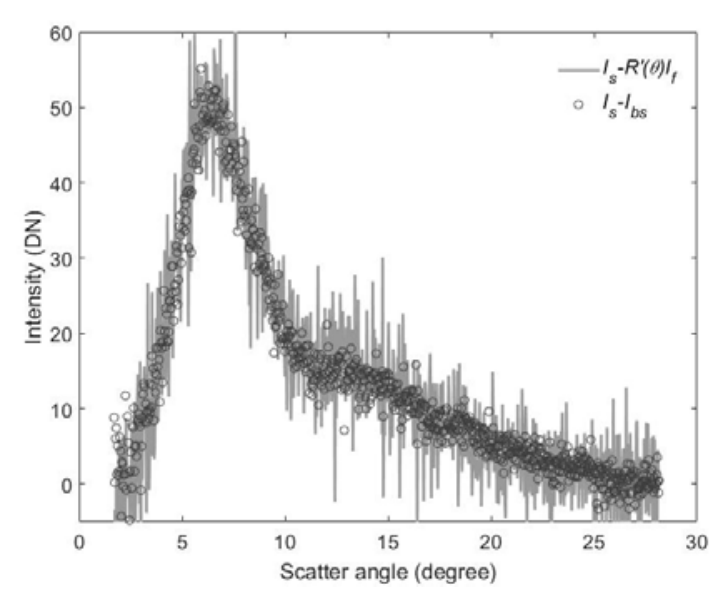

(b)

Figure 6. (a) Comparison of fitting curve $(1 / R(\theta))$ (obtained from $I_{f}(\theta) / I_{b s}(\theta)$ ) to the fitting curve $\left(1 / R^{\prime}(\theta)\right)$ (obtained from $I_{f}(\theta) / I_{S}(\theta)$ ) at scatter angles below $3^{\circ}$ and above $22^{\circ}$. A Gaussian function (as in (1)) was fitted to the partial data for $I_{f}(\theta) / I_{S}(\theta)$, where the scatter angle is less than $3^{\circ}$ and larger than $20^{\circ}$ in order to avoid the diffraction signal. The fitting curve of $I_{f}(\theta) / I_{S}(\theta)$ that was applied to these parts of the data is identical to the fitting curve of $I_{f}(\theta) / I_{b s}(\theta)$. Therefore, the transmission correction factor can be estimated by using the XRD pattern $I_{s}(\theta)$ and flat image $I_{f}(\theta)$ only. (b) Comparison of the diffraction patterns of Perspex when the adjusted flat background image is being subtracted.

This second method for estimating the background signal can also be used to correct the fluctuation of the X-ray flux. The output of the X-ray source is proportional to $I_{S}$ at low angles. Therefore, by using this method, we can also normalize the signal to reduce the influence of X-ray source fluctuations. As shown in figure 7, the three measurements of the XRD profile of Perspex differed from each other before this correction method was used. This difference disappeared after the flat image was adjusted. In figure 7 (a), the XRD signal at scatter angles of less than $6^{\circ}$ has negative values, as the background signal (raw flat image) being subtracted has a higher intensity than the XRD signal. The diffraction peak at the lower scatter angle was restored by subtracting the corrected flat image. It can also be seen that the peak at larger scatter angle $\left(15^{\circ}\right)$ in figure 7 (b) has a higher intensity compared to the results before correction was made in figure 7 (a) 


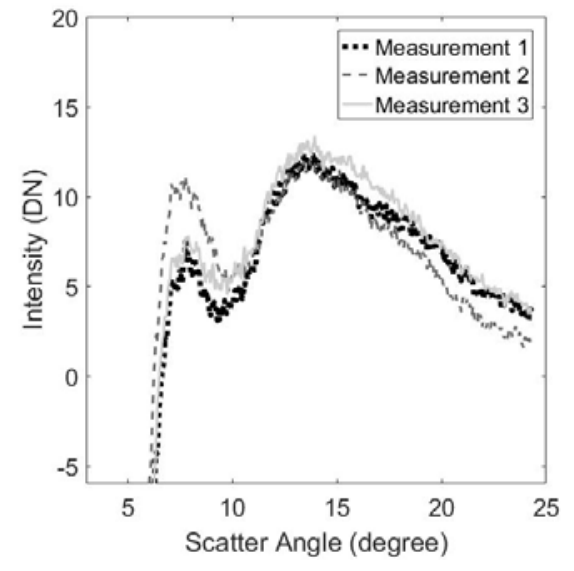

(a)

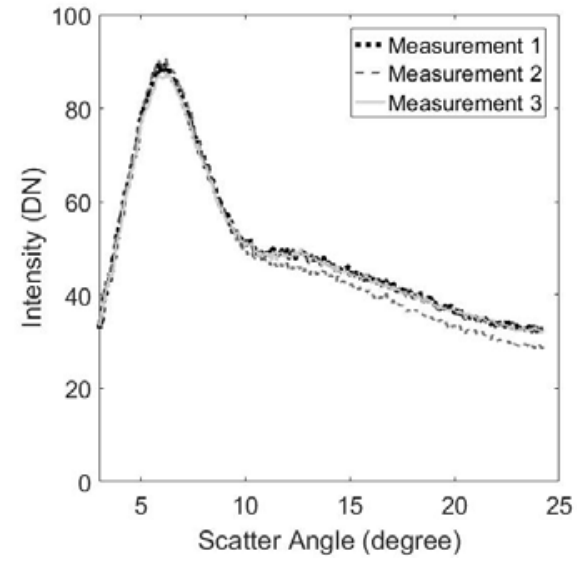

(b)

Figure 7. XRD profiles of Perspex. (a) The XRD data of Perspex, subtracting the flat image $I_{f}(\theta)$, and (b) XRD data of Perspex, subtracting the adjusted flat image $R(\theta) I_{f}(\theta)$.

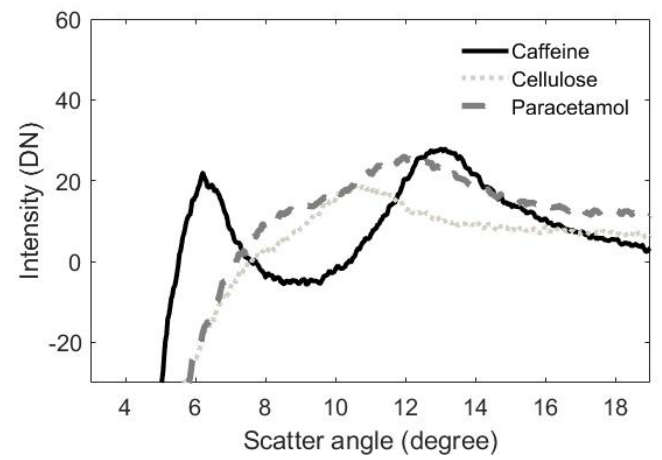

(a)

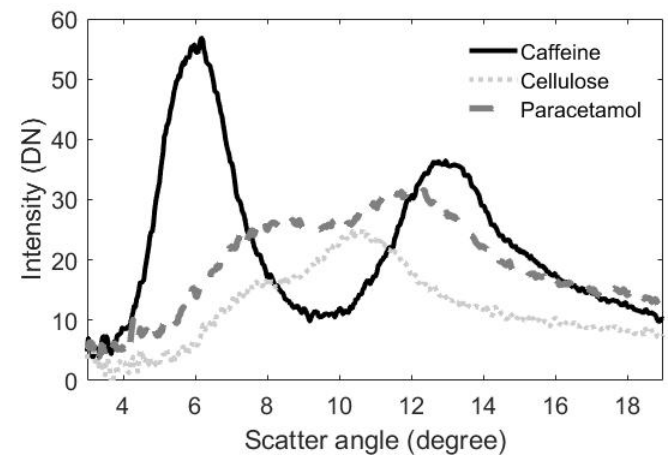

(b)

Figure 8. XRD pattern of other materials. (a) The XRD patterns from samples of $6 \mathrm{~mm}$ thickness of caffeine, cellulose and paracetamol, subtracting the flat image $I_{f}(\theta)$, and (b) XRD data subtracting the adjusted flat image $R(\theta) I_{f}(\theta)$.

This correction method can also be used for other materials. Figure 8 shows the diffraction patterns of $6 \mathrm{~mm}$ Caffeine, Cellulose and Paracetamol samples. Notice that these three materials have crystalline structure, but when they are in a powder form in a XRD system with polyenergetic radiation, the blurring due to the limit of angular and energy resolutions of the system make the XRD pattern more similar to one from an amorphous material, which makes them suitable to demonstrate an application of the proposed correction method.

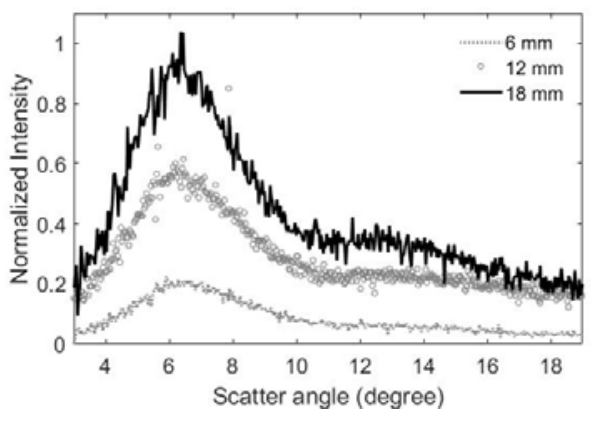

(a)

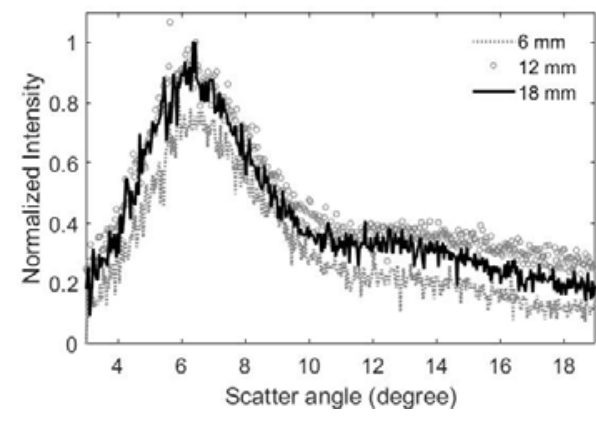

(b)

Figure 9. XRD profiles of Perspex with different thickness: (a) without and (b) with attenuation correction 
To investigate whether this correction method can also be used in different thickness of Perspex, same Perspex sample of three thickness (6,12 and $18 \mathrm{~mm}$ ) were imaged. Their diffraction patterns are extracted and plotted in figure 9 (a). The thickness of the sample will influence: (1) the total attenuation (including photoelectric absorption, Compton scatter and coherent scatter) of the signal; (2) the amount of coherent scatter. These two factors have opposite effects on the XRD signal intensity. A thicker sample creates more attenuation, which results in a reduction of intensity. However, an increase in thickness means an increase in the material that produces coherent scatter, which causes an increase in the coherent scatter intensity. As we can see from figure 8 (a), for these three samples, the decrease of coherent scatter signal due to the decrease of thickness outweighs the reduction of attenuation. This can be corrected by using the following equation:

$$
R_{\text {att }}(E)=e^{-\mu(E) t} \times\left(1-e^{-\mu_{c o h}(E) t}\right)
$$

where $R_{a t t}(E)$ is the correction factor for photon with energy $E, \mu$ refers to the linear attenuation coefficient of the material, $t$ is the thickness of the sample and $\mu_{c o h}$ is the linear coherent scatter coefficient.

As the energy spectrum in the experiment is dominant by Mo $\mathrm{K} \alpha$ line with a narrow width, the energy of Mo Ka $(17.4 \mathrm{keV})$ can be used to calculate the correction factor $R_{a t t}$. As shown in figure 9 (b), after dividing the ADXRD signal by the correction factors, the XRD patterns become very similar. The difference of the XRD profiles after correction in figure 9 (b) could due to either beam hardening: the thicker the sample, the higher energy of the X-rays beam passing through the sample or system error in different measurements.

\section{Summary}

This paper proposes two methods to remove the background for XRD signal from a large sample (more than $3 \mathrm{~mm}$ ) using a laboratory X-ray source (when a large collimator aperture is required). These two methods not only benefit the clinical use of XRD for cancer diagnosis, but can also be applied to extracting the XRD signal from bulk materials, such as those used for security screening. Both of these methods show agreement on the same data set. However, the second method has advantages in that: 1) it does not require the sample to be irradiated twice and 2) it can also reduce the influence of fluctuations between the acquisition of the flat field and that of the actual diffraction data in the radiation source beam intensity. 


\section{Reference}

Bohndiek S E 2008 Active pixel sensors for the breast biopsy analysis using x-ray diffraction Ph.D. thesis University College London London U.K.

Bohndiek S E, Cook E J, Arvanitis C D, Olivo A, Royle G J, Clark A T, Prydderch M L, Turchetta R and Speller R D 2008 A CMOS active pixel sensor system for laboratory- based x-ray diffraction studies of biological tissue Phys. Med. Biol. 53 655-72

Chaparian A, Oghabian M A, Changizi V 2012 Introducing an optimized method for obtaining x-ray diffraction patterns of biological tissues IJMP 8 9-17

Chaparian A, Oghabian M A, Changizi V and Farquharson M J 2010 The optimization of an energydispersive X-ray diffraction system for potential clinical application Appl. Radiat. Isot. 68 223745

He, B B 2009 Two-Dimensional X-Ray Diffraction (John Wiley \& Sons, NJ: Hoboken) p 375

Konstantinidis A, Zheng Y, Philip D, Vinnicombe S and Speller R 2012 A novel wafer-scale CMOS APS X-ray detector for breast cancer diagnosis using X-ray diffraction studies $J$. Instrum.JINST07(2012)C12005

Lewis R A et al 2003 X-ray refraction effects: Application to the imaging of biological tissues $\mathrm{Br}$. $J$. Radiol. 76 301-08

O’Flynn D, Reid C B, Christodoulou C, Wilson M D, Veale M C, Seller P, Hills D, Desai H, Wong B and Speller R 2013 Explosive detection using pixellated X-ray diffraction (PixD) J. Instrum. JINST08(2013)P03007

Pani S, Cook E J, Horrocks J A, Jones J L and Speller R D 2010 Characterization of breast tissue using energy-dispersive X-ray diffraction computed tomography Appl. Radiat. Isot. 68 1980-87

Petsche S J, Pinsky P M, 2013 The role of 3-D collagen organization in stromal elasticity: a model based on X-ray diffraction data and second harmonic-generated images. Biomech. Model. Mechanobiol. 12 1101-13

Podgorsak E B 2016 Radiation Physics for Medical Physicists (Springer, Germany: Berlin)

Poulsen H F 2004 Three-Dimensional X-Ray Diffraction Microscopy: Mapping Polycrystals and Their Dynamics (Springer, UK: Milton Keynes) pp 53

Sampath S, Isdebski T, Jenkins J E, Ayon J V, Henning R W, Orgel J P R O, Antipoa O and Yarger J L 2012. X-ray diffraction study of nanocrystalline and amorphous structure within major and minor ampullate dragline spider silks. Soft Matter 8 6713-22

Sidhu S, Falzon G, Hart S, Fox J G, Lewis R and Siu K K W 2011. Classification of breast tissue using a laboratory system for small-angle x-ray scattering (SAXS) Phys. Med. Biol. 56 6779-91

Sternberg R S 1983 Proc. IEEE Comput. Conf. 16 22-34

Theodorakou C and Farquharson M J 2009 The classification of secondary colorectal liver cancer in human biopsy samples using angular dispersive x-ray diffraction and multivariate analysis. Phys. Med. Biol. 54 (16) 4945-57

Zhao C, Konstantinidis A.C, Zheng Y, Anaxagoras T, Speller R.D, and Kanicki J $201550 \mu$ m pixel pitch wafer-scale CMOS active pixel sensor x-ray detector for digital breast tomosynthesis Phys. Med. Biol. 60 8977-9001

Zheng Y, Vassiljev N, Konstantinidis A, Griffiths J and Speller R 2015 Limit of Detection in X-ray Diffraction Measurements of Tissue Equivalent Samples J. Phys. Conf. Ser 637(2015)012037 